\title{
IDENTIFICATION OF ACTINOMYCETYES IN THE BIODEGRADED WALL MATERIAL BY PCR AMPLIFIED GENE FRAGMENT CODING FOR 16s rRNA
}

\author{
Garima Arya* and Purshotam Kaushik \\ Deptt. of Botany and Microbiology, Gurukul Kangri Vishwavidyalay., Hardwar \\ *Corresponding Author’s e-mail: garimaaryaphd@gmail.com, Webpage: purshotam.kaushik.googlepages.com
}

Received 17 Oct 2012; Review Completed 06 Nov 2012; Accepted 06 Nov 2012, Available online 15 Nov 2012

\begin{abstract}
Generally, old buildings and monuments are often affected by biodecay. An inventory of the existing microbial consortia which associated with the damage part of the buildings wall is not yet integral part of the restoration process. This stems from the lack of effective means for such stocktaking. Although, Actinomycetes, fungi and bacteria cause severe damage through mechanical process from the growth into wall and its grounding and through their metabolism. Physiological and molecular approach based techniques were used in the detection and identification of DNA sequences encoding rRNA to actinomycetes present on building wall. DNA from isolate was extracted and 194 base pair fragments of the 16S rDNA were amplified with cultured primers. The 16S rDNA fragments of uniform length obtained from the different species were separated according to their sequences difference by PCR. By the sequencing excised and reamplified individual DNA bands, characterized the phylogenetic tree of corresponding actinomycetes. Using this approach, we identified members of the genera of Streptomyces, Nocardia and Micromonospora.
\end{abstract}

Key words: Actinomycetes, Nocardia, Micromonospora, Streptomyces

\section{INTRODUCTION}

It is well recognized that the microorganisms can be responsible for the destruction of building material and cultural heritages including the decay of concrete, marble and sandstone $\mathrm{e}^{1,4}$. Thus, it is not surprising that the building material can also support the growth of microorganisms, which are responsible for the biodegradation of building material $^{3}$. Actinomycetes which, interalia, grow on the surface of the building wall might discolour the cemented or painted surface but also by excreting metabolic products. Thus, the colonization of external surfaces of buildings by microorganisms cause the aesthetically unacceptable appearance of wall surfaces by biogenic pigment ${ }^{2}$ and the production of extracellular polymeric substances that cause mechanical stress to the mineral structure due to shrinking and swelling cycles of these colloidal biogenic slimes inside the pore system. However, mycelia of the actinomycetes and fungi can penetrate into the paint layer and its grounding, resulting in the mechanical destruction of the cultural heritage and building.

DNA based techniques of identification of microorganisms have revealed that the conventional microbiological efforts to isolate actinomycetes present in building wall material. To obtain a better understanding of the microbial community and potential role of actinomycetes in the process of degradation of building material; we applied the molecular approach of PCR amplified gene fragments coding for 16S rRNA. The $16 \mathrm{~S}$ rDNA fragments of DNA of actinomycetes, which could be isolate from building wall material by conventional microbiological techniques were sequenced and analysed phylogenetically. Our results demonstrate that the molecular biological approach is a potential tool to identify the actinomycetes in biodegradation process.

\section{MATERIAL AND METHODS}

Sample collection: The samples were collected from different sites of building wall material at Hardwar region. The samples were taken from an area which showed a rosy and dirty swelling cover of various intensities by scrapping off surface material and wall plaster to a depth of 1-5 mm. The total sample was split into two parts. One part was used for isolation of aerobic and heterotrophic actinomycetes by standard cultivation techniques and the second part used for scanning electron microscopy.

Electron microscopy: Particles of the samples were mounted with aluminium stubs and sputter coated to a 3 $\mathrm{nm}$ depth with gold platinum (80:20), using a magnetic sputter coater. Electron micrography was performed with a Hitachi S-4100 scanning electron microscope.

Isolation of actinomycetes: The sample material from biodegraded wall was suspended into saline solution and shaken for 15 minutes to remove the intensities from the particles $^{5}$ and transferred to agar plates in dilution from $10^{-2}$ to $10^{-6}$. The following media were used: actinomycetes agar, nutrient agar, nutrient broth, glycerol asparagine agar, streptomycetes agar. The plates were incubated at $28^{\circ} \mathrm{C}$ for 3-7 days. The number of CFU was determined every 3 days. Isolation of well defined single colonies resulted in the isolation of twenty nine isolates as pure culture and three isolates out of them further selected for identification by $16 \mathrm{~S}$ rDNA sequencing.

DNA extraction: Genomic DNA from the isolated isolates of actinomycetes was obtained by chloroformphenol extraction ${ }^{8}$. Isolates were dissolved in TE buffer and subjected to three freeze-thaw cycle to lyse the isolates ${ }^{5}$. After centrifugation, $5 \mu$ l of the supernatant was used as DNA template in the PCR. The protocol for DNA 
extraction including microwave heating, freeze thawing, lysis solution were tested.

Sequencing of 16S rDNA fragments: DNA extracted ${ }^{8}$ from isolates of actinomycetes and DNA eluted from the excised DGGF bands were reamplified with the same forward primer (5'-CCTACGGGAGGCAGCAG-3') including an additional sequence extension (5'AAAATTAACCCTCACTAAAG-3') at its 5; end the reverse primer (5'-ATTACCGCGGCTGCTGG-3') with the additional sequence (5'AAATTCACACAGGAAACAG-3') at its 5' end to facilitate DNA sequence analysis. The newly obtained PCR fragments were purified ${ }^{7}$ and sequenced directly with a LI-COR model 4000L automatic sequencing system.

Identification of actinomycetes by comparative sequence analysis: The sequence obtained from the isolated actinomycetes and DGGE bands were send to the ribosomal database project ${ }^{\mathbf{8}}$ and to the EMBL nucleotide sequence database for comparison. The similarity rank tool of the RDP and the FASTA search option for the EMBL database were used to search for close evolutionary relatives. Prealigned sequences of representatives of several groups were obtained and used for the identification of similarities to test sequences.

\section{RESULTS AND DISSCUSION}

\section{Electron microscopy of biodegraded wall material}

The electron microscopy of a biodegraded wall sample, which was taken from the surface, revealed a dense microbial colonization of the material (Figure 1). The dominant colonizing microorganisms showed filamentous morphologies ranging in length from 4 to 15 um. The filaments not only were attached to the material but above grew with in the inter sticks of the material occasionally forming tuberous growths. Figure 2 suggests that the growth of these microorganisms resulted in a loosening of the consistency of the ground material.

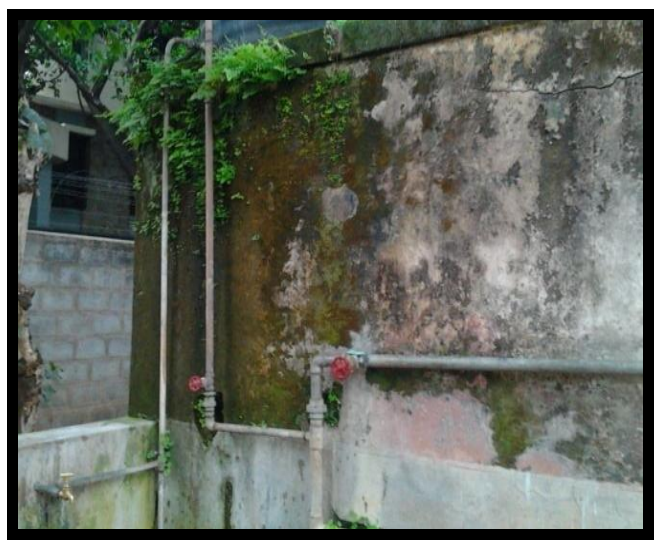

Figure 1: Microbial activities on domestic building at Haridwar (Uttarakhand)

Isolation of actinomycetes from building wall: As all the isolation process twenty nine isolates of actinomycetes were isolated. On the basis of morphological, physiological characteristic it was said to be isolates belong to actinomycetes family and the all isolates were classified into three groups. Three isolates $\left(\mathrm{A}_{11}, \mathrm{~A}_{20}, \mathrm{~A}_{27}\right)$ which shows better antimicrobial potential, were chosen for further study.

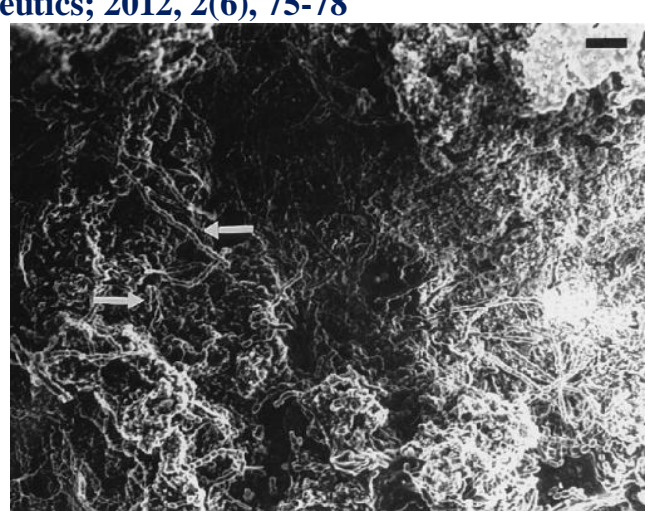

Figure 2: Electron micrograph of surface material of building sample. The Filamentous attached to the material are indicated by arrow.

Phylogentic classification of isolates of actinomycetes: The alignment of the nucleotide sequence ( 749 base pair) of actinomycetes isolates were done through matching with the $16 \mathrm{~S}$ rRNA reported gene sequence in the gene bank. The data base of national centre for biotechnological information (NCBI) basic local alignment search tool (BLAST) available at (www.ncbi-nlm-ningov) was used to compare the isolate strains with sequence of the reference species of the isolates $\mathrm{A}_{11}, \mathrm{~A}_{20}$ and $\mathrm{A}_{27}$ contained in genomic database bank and produced a ethidium bromide stained gel separation pattern of actinobacteria DNA fragment coding for $16 \mathrm{~S} r$ RNA (Figure 3). The Figure 3 represents the ethidium bromide-stained DGGE separation pattern of bacterial DNA fragment coding for the $16 \mathrm{~S}$ rRNA. Lanes 1 to 4 and 7 to 11 show the fragments obtained from actinomycetes isolates $\mathrm{A}_{11}$ (lane 2, 3 and7), $\mathrm{A}_{20}$ (lanes 1, 8 and 10), $\mathrm{A}_{27}$ (lanes 4, 9 and 11), and (lanes 5 and 6 , representing control DNA. A weak band was visible only in the original gel. The position of the band is marked.). Lanes 5

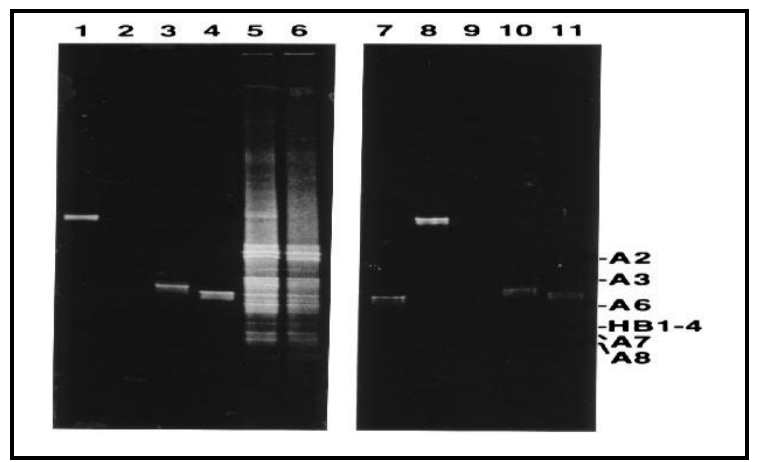

Figure 3: Gel Image of 16S rDNA amplicon

and 6 show the reproduced band patterns of the 16S rRNA genes which were amplified directly fromDNA. Bands A2, A3, A6, A7, and A8 were excised and sequenced.

The result exhibited similarity $91 \%$ with 10 strains from the uncultured known species of Streptomyces, Nocardia and Micromonospora. The 16S rRNA nucleotide sequence of Streptomyces, Nocardia and Micromonospora consisted of 749 bp each and the G-C content was $70 \%$. These results were in accordance to many authors who mentioned that the G-C content of the actinomycetes DNA is $69-78 \%^{7}$. Modern identification system are based on 16S rRNA sequence data, which have provided valuable information about test isolates $\left(A_{11}, A_{20}\right.$ and $\left.A_{27}\right)$ 
Garima et al

Journal of Drug Delivery \& Therapeutics; 2012, 2(6), 75-78

systematically and then have been used to identify several new isolates ${ }^{10}$. For data analysis, the phylogenetic tree in
Figure 4 was derived from the distance matrices using neighbor joining method ${ }^{12}$.

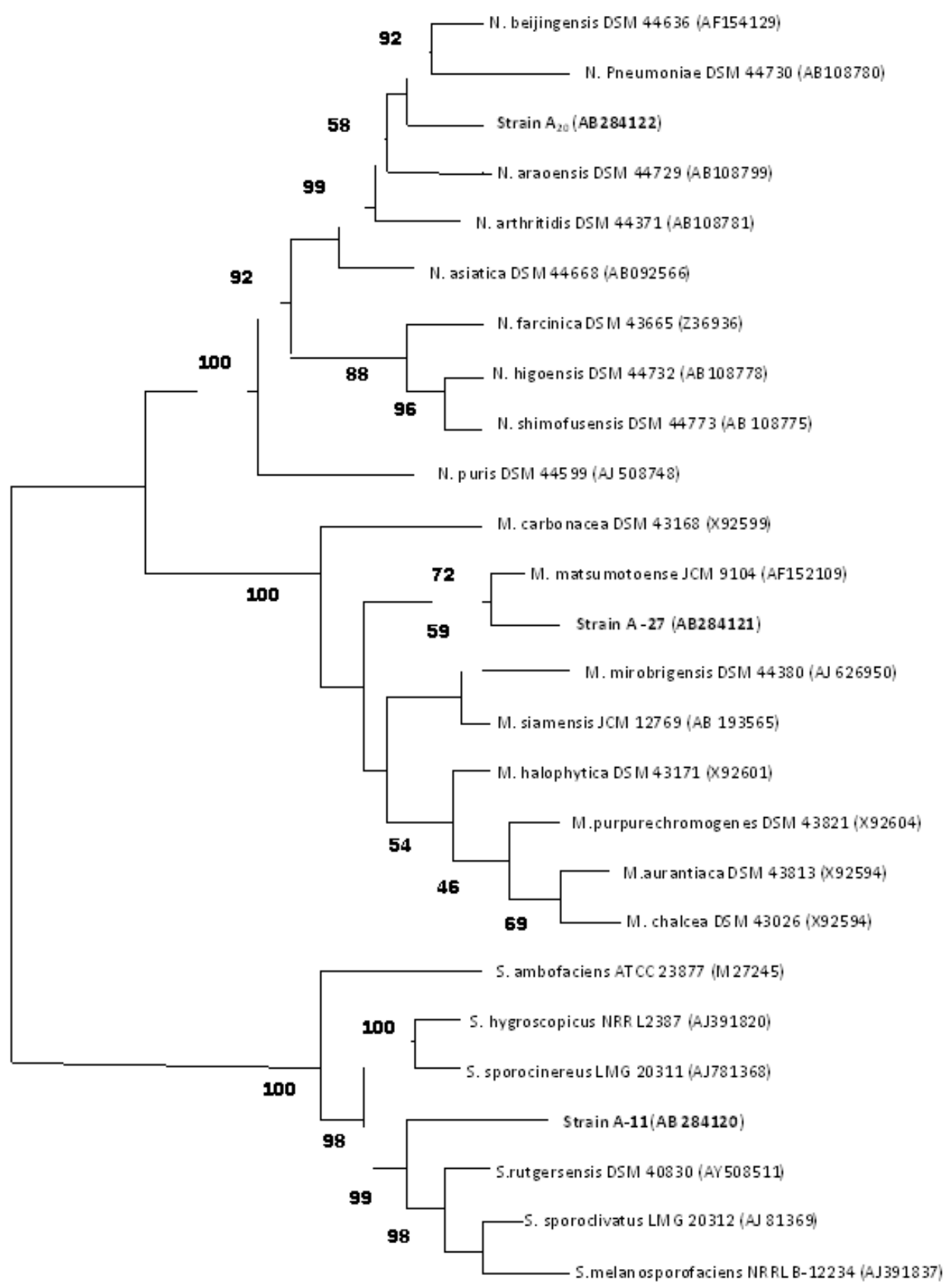

0.01

. Figure 4: Phylogenetic tree (evolutionary relationships of 26 taxa) 
Garima et al

Journal of Drug Delivery \& Therapeutics; 2012, 2(6), 75-78

A phylogenetic tree was constructed based on a $1216 \mathrm{bp}$ alignment of the cloned PCR amplicons and known actinomycete sequences (Figure 4). The actinomycete $16 \mathrm{~S}$ rDNA sequences amplified from DNA isolated from isolates of building walls affiliated with several clusters in the phylogenetic tree. The clones affiliated with three beanches, one including Nocardia beijingensis, Nocardia araoensis and Nocardia puris second include the Micromonospora carbonacea, Micromonospora chalcea and the third including Streptomyces ambofaciens, Streptomyces rutgersensis and Streptomyces melanosporofaciens. Other sequevars were also present; some of them being distantly related to sequences obtained from database. The isolate $\mathrm{A}_{11}$ closely related to Streptomyces rutgersensis and Streptomyces sporocinereus, isolate $\mathrm{A}_{27}$ closely linked to Micromonospora matsumotoense, Micromonospora siamensis and Micromonospora mirobrigensis and the third isolate linked to the known species of Nocardia araoensis, Nocardia pneumonia and Nocardia beijingensis. Based on maximum identity score first ten sequences were selected and aligned using multiple alignment software program Clustal W. Distance matrix was generated using RDP database and phylogenetic tree was constructed using MEGA4. Therefore, on the basis of sequences producing significant alignment isolates $A_{11}$, $\mathrm{A}_{20}$ and $\mathrm{A}_{27}$ were suggesting to be new genomic Sterptomyces, Nocardia and Micromonospora species and their accession number as under:

Strain $A_{11}$ : accession number (AB284120) strain is Streptomyces species.

Strain $\mathrm{A}_{20}$ : accession number (AB284122) strain is Nocardia species.

Strain $\mathrm{A}_{27}$ : accession number (AB284121) strain is Micromonospora species.

\section{CONCLUSION}

Actinomycetes contribute to the mechanical destruction of wall materials as a result of the production of mycelia, which grow into the building material and its grounding.

\section{REFERENCES}

1. Anderson AS, Wellington EH. The taxonomy of Streptomyces and related genera. International Journal of Systematic and Evolutionary Microbiology, 2001,51: 797-814

2. Bock E, Sand W, The microbiology of masonry biodeterioration Journal of Applied Bacteriology, 1993, 74:503-514.

3. Lyalikova NN, Petushkova YP, Role of microorganisms in the weathering of minerals in building stone of historical buildings. Journal of Geomicrobiology, 1991, 9:91-101.

4. Pearson, WR, Rapid and sensitive sequence comparison with FAST and FASTA. Methods of Enzymology, 1990, 183:63-98.

5. Petushkova YP, Lyalikova NN, Poglasova MN, Microorganisms discovered on the frescoes of the Ferapoint Monastery. Mikrobiologiya, 1989,58:1021-1030.

6. Reichlin-Minoretti A, Bindemittelanalysen an vorindustiellen malereien. Eidgenossische Technische Hochschule Zurich, 1983,15: 111-114.

7. Saiki, R K, Gelfand DH, Stoffel SJ. Scharf SJ, Higuchi R, Horn GT, Mullis KB, Erlich HA, Primer-directed enzymatic amplification of
By using conventional microbiological techniques, we could also isolate bacteria of this group. They were identified by $16 \mathrm{~S}$ rDNA sequence analysis. All twenty nine isolates are categories into three groups. Thirteen isolates are Gram-positive actinobacteria with high G-C content, i.e., the actinomycete lineage and belongs to the Streptomycetes species. Nine isolates of actinomycetes belong to Nocardia species and the seven isolates which categories on the basis of morphological, physio-chemical and molecular characteristics and seven isolates related to the Micromonospora. Although previously not identified on wall material, actinobacteria are ubiquitous aerobic, heterotrophic organisms. The kind of damage that actinobacteria can do to wall paints has not yet been clarified; however, since they can live on a wide variety of compounds, including aliphatic alcohols, some amino acids, decarboxylic and fatty acids, unbranched hydrocarbons and sugars, they may also use organic compounds, which are usually present in the paint layer of buildings wall ${ }^{3}$, as a carbon source. Future activities will focus on three aspects:

1. Sequencing of more bands from the gene sequences pattern to explore the microbial diversity in more detail,

2. Attempting to isolate bacteria related to PCR based $16 \mathrm{~S}$ rDNA sequences such as anaerobic and halotolerant bacteria, and

3. Determining the abundance of particular actinobacterial groups which have been found either by microbial isolation or by PCR based analysis ${ }^{11}$.

It is hoped that an integrated approach of molecular and microbiological techniques will lead to a better understanding of the composition of bacterial species on ancient wall materials and to new ways to restore and conserve this cultural heritage.

\section{ACKNOWLEDGEMENT}

Sincere thanks to Dr. Deepak Vashist, Sequencer Private Limited, Ahemdabad, for his help with molecular identification of isolates. We thank to Dr. N. K. Juyal and Dr. Khanna, SEM Laboratory Dehradun.

DNA with thermostable DNA polymerase. Science, 1988, 239:487491.

8. Saitou N, Nei M, The neighbor joining method: A new method for reconstructing phylogenetic trees. Molecular Biology and Evolution, 1987, 4: 406-425.

9. Takeuchi T, Sawada H, Tanaka F. and Matsuda I Phylogenetic analysis of actinomycetes based on 16S rRNA sequnces. International Journal of Systematic Bacteriology, 1986, 46: 476-479.

10. Tamura K. Dudley J. Nei M, Kumar S, MEGA4: Molecular evolutionary genetics analysis (MEGA) software version 4.0. Molecular Biology and Evolution, 2007, 24: 1596-1599.

11. Urzi C, Lisi S, Criseo G, Pernice A, Adhesion to and degradation of marble by a Micrococcus strain isolated from it. Geomicrobiol. J, 1991, 9:81-90.

12. Wagner MR, Erhart W, Manz R, Amann, H, Lemmer D, Schleifer KH. Development of a rRNA-targeted oligonucleotide probe specific for the genus actinetobacter and its application for in situ monitoring in activated sludge. Applied and Environmental Microbiology, 1994, 60 792-800. 